\title{
PERFORMANCES ZOOTECHNIQUES DE TRYONOMYS SWINDERIANUSEN CAPTIVITÉ EN ZONE D'ALTITUDE
}

\author{
ZOOTECHNICAL PERFORMANCES OF GRASSCUTTER \\ (TRYONOMYS SWINDERIANUS) IN CAPTIVITY IN SOUDANO-GUINEAN HIGHLANDS
}

\author{
Ngoula, F.*, F. Meutchieye, A. Kenfack, H.F. Defang, J. Awah-Ndukum, Z. Manfouo \\ and J. Tchoumboué
}

Université de Dschang. Faculté d'Agronomie et des Sciences Agricoles. Département des Productions Animales. B.P. 222 Dschang. Cameroun. *Correspondances: fngoula@yahoo.frou fngoula2004@yahoo.fr

\section{MotS CLÉS ADDITIONNELLES}

Gestation. Mise-bas. Mortinatalité. Sex-ratio. Morbidité. Sevrage. Mortalité.

\section{RÉSUMÉ}

Dans le but de contribuer à l'amélioration des performances de reproduction des aulacodes (Tryonomys swinderianus) en captivité, une étude a été menée en 2004 à la station de diffusion des aulacodes du CIPCRE ${ }^{1}$ à Bafoussam (ouestCameroun). Un total de 53 mises bas (37 en première et 16 en seconde) a été suivi pour les performances de reproduction. Les principaux résultats ont été les suivants : la durée de gestation a été de $158 \pm 6$ jours. La taille de la portée, de 4,44 $\pm 1,41$ petits en moyenne, a été plus élevée à la seconde mise-bas. Le taux de mortinatalité, de près de $7 \%$ à la première, était significativement plus élevé $(11 \%)$ à la seconde mise-bas. Le nombre de petits nés vivants par portée $(4,12 \pm$ $1,68)$ est resté relativement inchangé pour les deux mise-bas. Le sex-ratio, de 0,92/1 à la première, $a$ été plus élevé à la seconde mise-bas $(1,06 / 1)$. Le taux de mortalité post natal $(30 \%)$ a baissée avec le numéro de mise-bas, variant inversement au poids à la mise en reproduction des femelles. Le poids à la naissance et au sevrage $(138 \pm 21$ et $633 \pm 84 \mathrm{~g}$ respectivement) ont été plus élevé à la seconde mise bas, le taux de sevrage étant de $97 \%$. Les principales causes de morbidité et de mortalité ont été: les affections respiratoires, gastro-intestinales et les accidents, dont $70 \%$ des mortalités totales. Les maladies liées à l'alimentation

${ }^{1}$ ONG engagée dans le développement rural au Cameroun.

Recibido: 20-1-07. Aceptado: 17-9-08.

\section{AdDitional KeYWORDS}

Pregnancy. Birth. stillbirths. Sex-ratio. Morbidity. Weaning. Mortality.

ont été plus fréquentes chez les mâles que chez les femelles. Par contre, les reproductrices mouraient pratiquement deux fois plus que les reproducteurs.

\section{SUMMARY}

In order to improve on the reproductive performances of grasscutters (Tryonomys swinderianus) in captivity, a study was conducted in the grasscutter distribution centre at CIPCRE $^{1}$ Bafoussam, Cameroon. The reproductive performances of 53 gestating females (37 in the first and 16 in the second) were studied. The major results showed that the average litter size at first litter was $4.44 \pm 1.41$ which increased on second birth. A mortality rate of $7 \%$ was observed in the first litter which significantly $(p<0.05)$ increase to $11 \%$ in the second litter. The number of grasscutters born alive per litter $(4.12 \pm 1.68)$ remains relatively unchanged in the two litters. $A$ sex ratio of 0.92:1 and 1.06:1 was observed in the first and second litter respectively. The post natal mortality rate $(36 \%)$ decreased with litter number and varies inversely with the female weight at reproductive maturity. The birth and weaning weight $(138 \pm 21$ and $533 \pm 84 \mathrm{~g}$ respectively) were higher in the second litter with weaning rate of $97 \%$. The mortality rate of $70 \%$ was observed in the study which resulted from respiratory, gastro-intestinal tract diseases and some acci- 
dental cases. Metabolic diseases were more frequent in the males grasscutters. However, death rate in the females was twice that of the males.

\section{INTRODUCTION}

L'Afrique subsaharienne compte parmi les régions les plus sous-alimentées du monde (FAO, 2002), à cause d'une démographie galopante dont le taux d'accroissement est de $2,7 \%$ par an (World Bank, 2002). En effet, selon FAO (2002), 34\% soit le tiers de la population est atteint de malnutrition. En Afrique centrale, cette catégorie de la population est passée de $36 \%$ entre 1978 1981 à 37 et $50 \%$ entre $1990-1992$ et 1996 1998 respectivement (FAO, 2001). Si les besoins énergétiques sont relativement satisfaits, le déficit en protéines, plus particulièrement celles d'origine animale reste élevé à cause de l'insuffisance des productions animales conventionnelles.

Parmi les alternatives envisagées comme solution, figure l'élevage du gibier. Des essais sont en cours sur des espèces comme l'escargot géant (Achatina sp), le francolin (Francolinus sp), le crocodile du Nil (Crocodylus sp), l'antilope (Sylvicapra sp), le cricétome (Cricetomys sp), l'aulacode (Tryonomys swinderianus) etc.

L'aulacode, très apprécié des populations d'Afrique centrales et de l'ouest, fait l'objet d'un élevage expérimental depuis près de vingt ans (Siter et al., 1991). Plusieurs sources attestent qu'il est élevé avec succès dans des milieux paysans, notamment en Afrique de l'Ouest (Schrage et Yewadan, 1995).

D'introduction récente au Cameroun, les performances d'élevage n'y ont pas encore été évaluées. Or leur connaissance en rapport avec les facteurs qui les influencent est nécessaire pour mieux préciser les axes d'amélioration dans ces conditions particulières. Le présent travail a pour objectif de contribuer à une meilleure connaissance des performances de reproduction, et les principales causes de mortalité des aula- codes en captivité dans les conditions de l'Ouest du Cameroun.

\section{MATÉRIELETMÉTHODES}

\section{MILIEUd'ÉTUdE}

L'étude s'est déroulée entre Avril et Août 2004 à la station de diffusion des aulacodes du CIPCRE à Bafoussam, Ouest-Cameroun, (LN 5-6 ${ }^{\circ} 15, \mathrm{LE} 10-11^{\circ}$ ). L'altitude y oscille entre 1294 et $2740 \mathrm{~m}$. le climat est de type Soudano-guinéen tempéré par l'altitude, avec deux saisons: une saison pluvieuse de 8 mois (de mars à octobre) et une saison sèche de 4 mois (de novembre à février). La pluviométrie se situe entre 1300 et $1900 \mathrm{~mm}$ et la température de 20 à $25^{\circ} \mathrm{C}$ en moyenne. L'humidité relative est généralement supérieure à $60 \%$ et la végétation de type savane boisée d'altitude.

\section{TECHNIQUESD'ÉLEVAGE \\ Logement et alimentation}

Les aulacodes sont élevés dans des bâtiments en parpaings, bien aérés. Les aulacodères utilisés sont des enclos et des cages superposées avec des surfaces variant de 0,35 à $0,56 \mathrm{~m}^{2}$. La hauteur des loges est de $0,60 \mathrm{~m}$.

L'alimentation est basée sur le fourrage naturel (Pennisetum purpureum, Trypsacum laxum, Zea mays, Sheshum edulis), supplémenté avec un concentré à $25 \%$ de son de blé cubé et $75 \%$ de maïs grain. Par ailleurs, un os à croquer est servi comme supplément minéral et pour limer les incisives dont la croissance est continue chez l'aulacodes.

\section{Conduite de l'élevage}

Les animaux sont logés en groupes. Tous reçoivent les mêmes aliments. La distribution se fait en deux temps: fourrage-eau, puis supplément une à deux heures après. L'identification est faite dès la naissance. Les informations sont collectées sur des fiches et concernent la date, le numéro de cage et de la bête, le sexe, le poids, les 


\section{REPRODUCTION DES AULACODES EN CAPTIVITÉ}

observations ou opérations. L'observation des animaux est faite quotidiennement. Les pesées ont lieu à la naissance, au sevrage et à la mise en reproduction.

\section{Laprotection sanitaire}

Les mesures de protection sanitaires adoptées sont: l'utilisation du jus de citron comme anti-stress, la désinfection des enclos et cages, le déparasitage trimestriel et le traitement des blessures aux antibiotiques.

\section{Conduite de la reproduction}

Les reproducteurs sont conduits en groupes polygames ( 1 mâle pour 2 à 5 femelles). La mise en reproduction se fait à 6-7 mois et à $1500 \mathrm{~g}$ pour les femelles, à 7-8 mois d'âge et $2000 \mathrm{~g}$ pour les mâles. Le test de gestation utilisé est l'observation du mucus vaginal 45 jours après la mise au mâle et ensuite tous les 15 jours. Les gestantes sont séparées du groupe après le test et conduites dans des cages de gestation. Les mises-bas ont lieu dans des cages individuelles où les femelles sont transférées deux semaines environ avant la date présumée, déterminée à partir du test de gestation positif. Au sevrage à 42 jours, les petits sont transférés dans des cages d'engraissement.

Pour l'évaluation des performances de reproduction, nous avons exploité les fiches d'élevage. Les données ont porté sur 84 reproducteurs (22 mâles et 62 femelles) pour un total de 53 mises-bas dont 37 en première et 16 en seconde). L'exploitation des fiches a été complétée par des observations directes et des pesées à l'aide d'une balance de précision $25 \mathrm{~g}$.

\section{PARAMĖTRESÉTUDIÉS}

Les paramètres étudies étaient:

- durée de gestation (jours) $=$ date de mise bas-date de test positif +40 jours.

- taille de la portée $=$ nombre de petits à la naissance.

-taux de mortinatalité $(\%)=$ nombre de mort-nés/nombre de petits nés vivants $\mathrm{x}$ 100 .
- nombre de petits nés vivants par portée $=$ nombre total des nés vivants/nombre de mises-bas.

- sexe $\operatorname{ratio}(\mathrm{M} / \mathrm{F})$ à la naissance $=$ nombre de mâles nés vivants/nombre de femelles nées vivantes.

- taux de mortalité pré sevrage $(\%)=$ nombre de petits nés vivants-nombre de petits sevrés/nombre de petits nés vivants x 100 .

- taux de sevrage $(\%)=$ nombre de petits sevrés/nombre de petits nés vivants $\mathrm{x} 100$.

- sexe ratio $(M / F)$ au sevrage $=$ nombre de mâles sevrés/nombre de femelles sevrées.

- taux de mortalité post-sevrage $(\%)=$ nombre de petits morts entre 42 et 180 jours/ nombre de petits nés vivants $\mathrm{x} 100$.

- intervalle entre mises-bas (jours) $=$ date de la dernière-date de la précédente misebas.

- taux de morbidité $(\%)=$ nombre de cas présentant une affection/ nombre total des malades x 100.

- taux de mortalité $(\%)=$ nombre de morts d'une catégorie donnée/nombre total de morts $\mathrm{x} 100$.

\section{ANALYSE STATISTIQUE}

La statistique descriptive, et le test de Student ont été utilisés pour comparer et séparer les valeurs. La limite de signification était de $\mathrm{p}<0,05$.

\section{RÉSULTATS}

Les performances de reproduction des aulacodes en captivité sont résumées au le tableau I.

DuRÉE DE GESTATION, TAILLEDELAPORTÉE ETTAUXDEMORTINATALITÉ

La durée de gestation et la taille de la portée augmentent de façon non significative ( $p>0,05)$ à la seconde mise-bas. Par ailleurs, le taux de mortinatalité est significativement $(\mathrm{p}<0,05)$ plus élevé en deuxième mise-bas.

\section{NOMBREDEPETITSNÉS VIVANTS}

Indépendamment du sexe et du numéro 
NGOULA ETAL.

Tableau I. Evolution des performances de reproduction des aulacodes en fonction du numéro de mise-bas. (Evolution of the reproductive performances of grasscutters according to the number of parturition).

\begin{tabular}{|c|c|c|c|c|c|c|}
\hline Numéro de mise-bas & 1 & $\mathrm{n}$ & 2 & $\mathrm{n}$ & Moyenne & $\mathrm{n}$ \\
\hline Durée de gestation (j) & $158 \pm 6^{\mathrm{a}}$ & 34 & $157 \pm 11^{\mathrm{a}}$ & 16 & $158 \pm 8$ & 50 \\
\hline Taille de la portée & $4,39 \pm 1,25^{a}$ & 37 & $4,56 \pm 1,77^{a}$ & 16 & $4,44 \pm 1,41$ & 53 \\
\hline Taux de mortinatalité & $6,96^{\mathrm{a}}$ & 158 & $10,61^{b}$ & 66 & 7,59 & 224 \\
\hline \multicolumn{7}{|c|}{ Nombre de petits nés vivants par portée } \\
\hline mâles & $1,97 \pm 1,37^{a}$ & 37 & $2,13 \pm 1,17^{b}$ & 16 & $2,02 \pm 1,31^{a}$ & 53 \\
\hline femelles & $2,13 \pm 1,12^{\mathrm{a}}$ & 37 & $2,00 \pm 1,17^{b}$ & 16 & $2,05 \pm 1,14^{\mathrm{a}}$ & 53 \\
\hline mâles et femelles & $4,11 \pm 1,57^{a}$ & 37 & $4,13 \pm 1,93^{a}$ & 16 & $4,12 \pm 1,67$ & 53 \\
\hline Sexe ratio a la naissance (M/F) & $0,92 / 1^{\mathrm{a}}$ & 158 & $1,06 / 1^{b}$ & 66 & $0,96 / 1$ & 224 \\
\hline \multicolumn{7}{|l|}{ Poids à la naissance $(\mathrm{g})$} \\
\hline mâles & $136 \pm 19^{a}$ & 73 & $151 \pm 29$ & 34 & $141 \pm 22^{a}$ & 107 \\
\hline femelles & $129 \pm 22^{a}$ & 79 & $137 \pm 27^{a}$ & 32 & $130 \pm 23^{a}$ & 111 \\
\hline mâles et femelles & $134 \pm 19^{a}$ & 152 & $147 \pm 27^{a}$ & 66 & $138 \pm 21$ & 218 \\
\hline \multicolumn{7}{|c|}{ Nombre de petits sevrés par portée à 42 j } \\
\hline mâles & $1,83 \pm 1,23^{a}$ & 36 & $2,00 \pm 1,17^{b}$ & 14 & $1,96 \pm 1,18^{a}$ & 50 \\
\hline femelles & $2,14 \pm 1,24^{a}$ & 36 & $2,00 \pm 1,10^{b}$ & 66 & $2,08 \pm 1,22^{\mathrm{a}}$ & 52 \\
\hline mâles et femelles & $3,94 \pm 1,5^{a}$ & 36 & $4,00 \pm 1,75^{a}$ & 16 & $3,96 \pm 1,65$ & 52 \\
\hline Taux de sevrage/nés vivants (\%) & $97,41^{\mathrm{a}}$ & 158 & 97,00 & 66 & 97,32 & 224 \\
\hline Sexe ratio au sevrage (M/F) & $0,92 / 1^{\mathrm{a}}$ & 154 & $1 / 1^{b}$ & 64 & $0,94 / 1$ & 218 \\
\hline \multicolumn{7}{|l|}{ Mortalité au sevrage/nés vivants (\%) } \\
\hline mâles & $2,63^{a}$ & 76 & $5,88^{b}$ & 34 & $3,64^{a}$ & 110 \\
\hline femelles & $2,44^{a}$ & 82 & $0,00^{\mathrm{b}}$ & 32 & $1,75^{b}$ & 114 \\
\hline mâles et femelles & $2,53^{a}$ & 76 & $3,00^{\mathrm{a}}$ & 66 & 2,68 & 106 \\
\hline \multicolumn{7}{|l|}{ Poids au sevrage (g) } \\
\hline mâles & $536 \pm 79^{a}$ & 74 & $651 \pm 95^{b}$ & 32 & $567 \pm 98^{a}$ & 106 \\
\hline femelles & $504 \pm 75$ & 80 & $609 \pm 55^{b}$ & 32 & $533 \pm 84^{a}$ & 112 \\
\hline mâles et femelles & $519 \pm 68^{a}$ & 154 & $629 \pm 74^{b}$ & 64 & $549 \pm 85$ & 218 \\
\hline \multicolumn{7}{|c|}{ Mortalité post sevrage/nés vivants (\%) } \\
\hline mâles & $7,89^{a}$ & 76 & $0,00^{\mathrm{b}}$ & 34 & $5,45^{a}$ & 110 \\
\hline femelles & $3,66^{a}$ & 82 & $0,00^{\mathrm{b}}$ & 32 & $2,63^{b}$ & 114 \\
\hline mâles et femelles & $5,70^{a}$ & 158 & $0,00^{\mathrm{b}}$ & 66 & 4,02 & 224 \\
\hline
\end{tabular}

$(a, b)$ sur la même ligne et dans la même colonne, les valeurs affectées d'une même lettre ne diffèrent pas significativement $(p>0,05)$; effectif des animaux.

de mise-bas, le nombre de petits nés vivants par portée est de 4,12 $\pm 1,67$. Lorsqu'on considère le numéro de mise-bas indépendamment du sexe, il est non significativement $(\mathrm{p}>0,05)$ plus élevé à la seconde mise bas. A sexe égal, le nombre de mâles nés vivants augmente significativement $(\mathrm{p}<0,05)$ à la seconde mise-bas. L'inverse est relevé pour le nombre de femelles nées vivantes. A numéro de mise-bas égale, les mâles nés vivants par portée sont non significativement $(p>0,05)$ plus nombreux que les femelles en première mise bas. L'inverse est enregistré à la seconde mise-bas.

\section{PoIDS ÀlanAISSANCE}

Indépendamment du numéro de misebas, le poids des aulacodes à la naissance est de $7,80 \%$ plus élevé chez les mâles que chez les femelles. Cette différence n'est 


\section{REPRODUCTION DES AULACODES EN CAPTIVITÉ}

toutefois pas significative $(\mathrm{p}>0,05)$. Lorsqu'on considère le numéro de mise-bas, indépendamment du sexe, le poids à la naissance est de $8,84 \%$ plus élevé à la seconde mise-bas; bien que cette différence ne soit pas significative $(\mathrm{p}>0,05)$.

Quelque soit le sexe, le poids des aulacodeaux à la naissance est plus élevé (de 9,93\% chez les mâles, et 5,84\% chez les femelles) à la deuxième mise-bas. Toutefois, l'analyse statistique ne révèle de différence significative $(\mathrm{p}<0,05)$ que pour la valeur de ce paramètre enregistrée chez les mâles.

A numéro de mise-bas égal le poids à la naissance des mâles dépasse celui des femelles de 5 et $9,27 \%$ à la première et à la seconde mise-bas respectivement. Aucune différence significative $(p>0,05)$ n'est cependant trouvée.

\section{SeX-RATIOÀ lANAISSANCE (M/F) ET NOM- BREDEPETITSSEVRÉS}

Le sexe ratio à la naissance est de $13,21 \%$ significativement $(\mathrm{p}<0,05)$ plus élevé à la deuxième mise-bas. Par ailleurs, Indépendamment du numéro de mise bas, le nombre de mâle sevrés par portée est de $6 \%$ non significativement $(p>0,05)$ inférieure à celui des femelles. Lorsqu'on considère le numéro de mise-bas, et indépendamment du sexe, le nombre de petits sevrés par portée à la deuxième dépasse de $1,5 \%$ celui de la première mise-bas ; bien qu'aucune différence significative $(\mathrm{p}>0,05)$ ne soit relevée. A sexe égal, il augmente significativement $(p>0,05)$ de $9 \%$ chez les mâles à la seconde mise-bas à l'inverse des femelles. A numéro de mise-bas égal, il est de $17 \%$ significativement $(p<0,05)$ plus faible chez les mâles que chez les femelles en première et égal en seconde mise-bas.

\section{TAUXDESEVRAgE ETSEX-RATIO(M/F)AU SEVRAGE}

Le taux de sevrage (de $0,47 \%$ ) baisse de manière non significative $(\mathrm{p}>0,05)$ à la seconde mise bas. Le sexe ratio au sevrage est de $9 \%$ significativement $(p<0,05)$ plus élevé à la seconde mise bas.

\section{MORTALITÉPRÉ SEVRAGE}

Indépendamment du numéro de mise bas, le taux de mortalité pré sevrage est significativement $(p<0,05)$ deux fois plus élevé pour les mâles que pour les femelles. Lorsqu'on considère le numéro de mise bas, indépendamment du sexe, le taux de mortalités avant sevrage augmente de manière non significative $(\mathrm{p}>0,05)$ à la seconde mise-bas. A sexe égal il est significativement $(\mathrm{p}<0,05)$ plus élevé chez les mâles et plus faible pour les femelles en seconde mise bas. A numéro de mise-bas égal, le taux de mortalité pré-sevrage est plus élevé chez les mâles que chez les femelles tant en première qu'en seconde misebas. Cependant, cette différence n'est significative $(p<0,05)$ qu'en seconde mise-bas.

\section{Poids ausevrage}

Indépendamment du numéro de misebas, le poids au sevrage des mâles est non significativement $(p>0,05)$ supérieur (de $6 \%$ ) à celui des femelles. Lorsqu'on tient compte du numéro de mise-bas quelque soit le sexe, le poids au sevrage est significativement $(\mathrm{p}<0,05)$ plus élevé à la seconde mise-bas. Par ailleurs, il est non significativement ( $p>0,05)$ plus élevé chez les mâles quelque soit le numéro de mise-bas.

\section{MORTALITÉ POSTSEVRAGE}

Indépendamment du numéro de misebas, le taux de mortalité post sevrage est significativement $(p<0,05)$ deux fois plus élevé pour les mâles que les femelles. Lorsqu'on tient compte du numéro de misebas, indépendamment du sexe, le taux de mortalité post sevrage est significativement $(\mathrm{p}<0,05)$ plus grand à la premier mise-bas comparée à la seconde. A numéro de misebas égal, il est significativement $(\mathrm{p}<0,05)$ deux fois plus élevé pour les mâles que pour les femelles à la première mise-bas. Tandis qu'en seconde mise-bas, aucune mortalité n'est enregistrée après le sevrage. 
Tableau II. Importance des principales maladies rencontrées à la ferme. (Importance of the main diseases found in the farm).

\begin{tabular}{lc}
\hline Pathologies & $\%$ \\
\hline Affections respiratoires & 33 \\
Accidents & 20 \\
Indéterminées & 16 \\
Affections gastro-intestinales & 20 \\
Paralysies & 4 \\
Inappétence & 2 \\
Abcès & 2 \\
Dystocies & 2 \\
Hémorragies & 1 \\
Total & 100 \\
\hline
\end{tabular}

\section{MORBIDTÉ}

Les principales maladies rencontrées sont résumées dans le tableau II. Les plus fréquentes sont: les affections respiratoires, les problèmes gastro-intestinaux et les accidents, représentant $70 \%$ des pathologies des aulacodes. La proportion représentée par les maladies dont les causes sont indéterminées n'est pas négligeable (16\%).

\section{MORTALITÉS}

Les mortalités des aulacodes en fonction des causes, du groupe d'âge et du poids de mise en reproduction des femelles sont résumées aux tableaux III et IV.

\section{RÉPARTITIONDES MORTALITÉSENFONCTION DESCAUSES}

Indépendamment du sexe et de la tranche d'âge, les principales causes de mortalités sont par ordre d'importance les affections gastro-intestinales, les maladies respiratoires et les causes indéterminées représentant tous les trois $80 \%$ des mortalités totales.

\section{MoRTALITÉENFONCTIONDUGROUPED'ÂGE \\ Lorsqu'on prend en compte le groupe} d'âge:

\section{Les adultes}

Indépendamment du sexe et des causes, la mortalité des adultes représentent près des deux tiers des mortalités totales. En

Tableau III. Mortalités (\%) en fonction des causes, de la tranche d'âge et du sexe. (Mortality (\%) with respect to causes, age and sex).

\begin{tabular}{|c|c|c|c|c|c|c|c|c|c|c|c|c|}
\hline \multirow{2}{*}{ Causes } & \multicolumn{3}{|c|}{ Adulte $(>6)$} & \multicolumn{3}{|c|}{$\begin{array}{l}\text { Tranche d'âge (mois) } \\
\text { Subadulte }(1,5-6)\end{array}$} & \multirow{2}{*}{$\begin{array}{c}\text { Non } \\
\text { m }\end{array}$} & \multicolumn{2}{|c|}{ sevrés $(0-1,5)$} & \multicolumn{3}{|c|}{ Total/Mortalité totale } \\
\hline & $\mathrm{m}$ & $\mathrm{f}$ & $\mathrm{mf}$ & $\mathrm{m}$ & $\mathrm{f}$ & $\mathrm{mf}$ & & $f$ & $\mathrm{mf}$ & $\mathrm{m}$ & $f$ & $\mathrm{mf}$ \\
\hline$A R$ & 21,74 & 13,04 & 34,78 & 0,00 & 0,00 & 0,00 & 0,00 & 0,00 & 0,00 & 12,82 & 7,69 & 20,51 \\
\hline $\mathrm{n}$ & 5 & 3 & 8 & 0 & 0 & 0 & 0 & 0 & 0 & 5 & 3 & 8 \\
\hline$A$ & 4,35 & 17,39 & 21,74 & 7,69 & 0,00 & 7,69 & 0,00 & 0,00 & 0,00 & 5,13 & 10,26 & 15,39 \\
\hline $\mathrm{n}$ & 1 & 4 & 5 & 1 & 0 & 1 & 0 & 0 & 0 & 2 & 4 & 6 \\
\hline AGI & 4,35 & 8,70 & 13,04 & 38,46 & 23,08 & 61,54 & 66,67 & 33,33 & 100,00 & 20,51 & 15,38 & 35,89 \\
\hline $\mathrm{n}$ & 1 & 2 & 3 & 5 & 3 & 8 & 2 & 1 & 3 & 8 & 6 & 14 \\
\hline D & - & 8,70 & 8,70 & - & - & - & - & - & - & - & 5,13 & 5,13 \\
\hline$n$ & - & 2 & 2 & - & - & - & - & - & - & - & 2 & 2 \\
\hline I & 4,35 & 17,39 & 21,74 & 7,69 & 23,08 & 30,77 & 0,00 & 0,00 & 0,00 & 5,13 & 17,95 & 23,08 \\
\hline In & 1 & 15 & 5 & 1 & 3 & 4 & 0 & 0 & 0 & 2 & 7 & 9 \\
\hline Total & 20,51 & 38,46 & 58,97 & 17,95 & 15,38 & 33,33 & 5,30 & 2,56 & 7,69 & 43,59 & 56,41 & 100,00 \\
\hline $\mathrm{n}$ & 8 & 15 & 23 & 7 & 6 & 13 & 2 & 1 & 3 & 17 & 22 & 39 \\
\hline
\end{tabular}

$m=$ mâles; $f=$ femelles; $n=$ nombre d'observations; $A R=$ Affections respiratoires; $A=A c c i d e n t s ; A G I=$ Affections gastro-intestinales; $D=$ Dystocies; I= Indéterminées. 
Tableau IV. Effet du poids de mise en reproduction des femelles sur la mortalité (\%) des aulacodes en captivité. (Effect of reproductive weight of females on morality of grasscutters under captivity).

\begin{tabular}{lcccc}
\hline & $p<1500$ & $\begin{array}{c}\text { Tranches de poids }(\mathrm{g}) \\
1500<\mathrm{p}<2000\end{array}$ & $\mathrm{p}>2000$ & $\begin{array}{r}\text { Total/total } \\
\text { né vivant }\end{array}$ \\
\hline $\mathrm{n}$ & 16 & 133 & 70 & 219 \\
Mort-nés & $0,00^{\mathrm{a}}$ & $9,02^{\mathrm{b}}$ & $4,29^{\mathrm{a}}$ & 6,85 \\
Mortalités pré sevrage & $12,50^{\mathrm{a}}$ & $3,75^{\mathrm{b}}$ & $0,00^{\mathrm{c}}$ & 3,20 \\
Mortalités post sevrage & $18,75^{\mathrm{a}}$ & $3,75^{\mathrm{b}}$ & $4,29^{\mathrm{b}}$ & 5,02 \\
Total mortalité post-natale & $31,25^{\mathrm{a}}$ & $7,52^{\mathrm{b}}$ & $4,29^{\mathrm{b}}$ & 8,22 \\
Total & $31,25^{\mathrm{a}}$ & $16,54^{\mathrm{b}}$ & $8,57^{\mathrm{c}}$ & 15,07 \\
\hline
\end{tabular}

$\mathrm{n}=$ nombre d'observations.

${ }^{a b c}$ sur la même ligne, les valeurs affectées de la même lettre ne différent pas significativement $(p>0,05)$.

prenant en compte le sexe, les femelles, avec plus de $65 \%$ des mortalités adultes, sont plus affectées que les mâles. En considérant les causes, et indépendamment du sexe, les plus fréquentes sont les affections respiratoires, les accidents et les causes indéterminées, soit près de $80 \%$ des mortalités adultes. A causes égales, les mâles succombent plus aux affections respiratoires que les femelles. Par contre les femelles meurent trois fois plus d'accident et des causes indéterminées, et deux fois plus d'affections gastro-intestinales que les mâles.

\section{Les subadultes}

Lorsqu'on ne tient pas compte du sexe et des causes, la mortalité des subadultes représente environ le tiers des mortalités totales. En tenant compte du sexe indépendamment des causes, les mâles sont légèrement plus affectés avec environ $55 \%$ des cas. En considérant uniquement les causes, les subadultes meurent à plus de $90 \%$ d'affections gastro-intestinales et de causes indéterminées. A cause égale, les mâles subadultes succombent $70 \%$ plus d'affections gastro-intestinales que les femelles. Par contre, les femelles meurent trois fois plus d'affections indéterminées que les mâles.

\section{Les non sevrés}

Indépendamment du sexe et des causes, les petits en lactation ont enregistré moins de 10\% des mortalités totales. En considérant le sexe, les mâles en lactation meurent deux fois plus que les femelles. En tenant compte des causes, les problèmes gastro-intestinaux semblent être les seules causes de mortalités dans ce groupe d'âge.

\section{MORTALITÉENFONCTIONDUPOIDSDEMISEEN REPRODUCTIONDESFEMELLES \\ Indépendamment du groupe physio-} logique, la mortalité frappe deux fois plus les femelles mises en reproduction à moins de $1500 \mathrm{~g}$ que les reproductrices mises en activité entre 1500 et 2000 g. Elle baisse encore de moitié lorsque l'entrée en activité à lieu au-delà de $2000 \mathrm{~g}$ de poids. Ces différences sont significatives $(p<0,05)$. En tenant compte $\mathrm{du}$ groupe physiologique des petits, les mortalités pré et post-sevrage représentent plus de $30 \%$ des nés vivants pour les femelles ayant débutées leur carrière de reproductrice à moins de $1500 \mathrm{~g}$ de poids vif. Par contre, entre 1500 et 2000 g, la mortinatalité augmente paradoxalement de 0 à $9 \%$ alors que la mortalité prés et postsevrage baissent significativement $(p<0,05)$ et respectivement de 70 et $80 \%$. Au-delà de $2000 \mathrm{~g}$ de poids de mise en reproduction, la 
mortinatalité baisse encore de moitié et significativement $(\mathrm{p}<0,05)$ par rapport au groupe précèdent pendant que la mortalité post sevrage amorce une remontée non significative $(\mathrm{p}>0,05)$ de $0,54 \%$.

\section{DISCUSSION}

Il ressort de cette étude que la durée de gestation est de 2 et 6 jours plus élevés que celles trouvées par Van de Velde (1991) puis Siter et al. (1991) respectivement. Elle serait influencée par l'âge et la taille de la portée et devrait être plus courte chez les primipares et en cas de portée nombreuse (Deblay, 2002). Le taux de mortinatalité se situe dans les limites déterminées par Nguema et Edderai (2000). Il est cinq fois plus élevé que celui relevé par Schrage et Yewadan (1995).

La taille de la portée est de 15 et 5,22\% plus élevé que celles enregistrées part Schrage et Yewadan (1995) et Siter et al. (1991) respectivement à la première misebas. Elle se situe dans les limites trouvées par Mensah (2000). Elle serait influencée par des facteurs tels que la souche, l'âge et le poids de mise en reproduction des femelles. Le nombre de petits nés vivants par portée est resté relativement constant malgré le taux de mortinatalité plus élevé à la seconde mise-bas, du fait de l'augmentation de la taille de la portée. Le poids à la naissance, indépendamment du sexe, est proche de celui trouvé par Schrage et Yewadan (1995). Il serait corrélé positivement au poids de mise en reproduction de l'aulacodine et influencée par la qualité des aliments consommés pendant la gestation.

Le sex-ratio à la naissance observé dans nos résultats reste dans les limites trouvées par Mensah (2000) au Bénin. Il a augmenté significativement de $15 \%$ à la seconde misebas. Le poids à la naissance est supérieur de $9 \%$ à la valeur rapportée par Schrage et Yewadan (1995). Par contre, quelque soit le sexe, il est comparable à celui trouvé par Nguema et Edderai (2000).

Le taux de sevrage a légèrement diminué à la seconde mise-bas. Ceci serait imputable à l'augmentation de la taille de la portée entraînant une mortalité prés sevrage plus élevée pour des sujets à faible poids à la naissance. Par ailleurs, le sex-ratio au sevrage est resté très proche de l'unité, attestant d'une mortalité au sevrage peut influencée par le sexe de l'animal.

La mortalité pré sevrage a été faible pour tous les sexes. Elle représentait la moitié de celle trouvée par Nguema et Edderai (2000) et le quart de celles relevées par le CIRAD (2003). Il s'agirait d'un effet de l'âge et du poids à la mise en reproduction des géniteurs.

Le poids au sevrage a été de $5 \%$ supérieur à celui rapporté par Schrage et Yewadan (1995). Mais de 2\% inférieur à celui trouvé par Nguema et Edderai (2000). Il est plus élevé chez les mâles par rapport aux femelles. Ceci est en accord avec les travaux de Mensah et Ekue (2003) qui relèvent un dimorphisme sexuel en faveur des premiers, caractérisé entre autres par des gains de poids plus élevés. Ce poids augmente avec le numéro de mise-bas.

La mortalité post-sevrage est de 23\% plus faible que celle observée par Nguema et Edderai (2000). Elle représente les deux tiers des valeurs trouvées par le CIRAD (2003). Par ailleurs elle baisse significativement à la seconde mise-bas. Les causes de morbidité et de mortalité sont multiples. Les plus importantes sont les maladies respiratoires et les affections gastro-intestinales. Mensah et Ekue (2003) estiment également que les principales causes de morbidités et de mortalités chez les aulacodes sont liées aux infections à Staphyloccocus aureus, à Clostridium perfiengens et Corynebacterium sp. Provoquant des gastro-entérites et des maladies respiratoires. De plus, Schrage et Yewadan (1995) comparent le tube digestif de l'aulacode à celui du cheval du point de vue de la délicatesse.

Les accidents font partie des principales causes de mortalités enregistrées dans notre 


\section{REPRODUCTION DES AULACODES EN CAPTIVITÉ}

étude. Ceci pourrait s'expliquer par le fait que l'aulacode est un animal très craintif et s'expose à des accidents mortels tels que évoqués par Van de Velde (1991). La baisse de la mortalité des petits avec l'augmentation de l'âge de mise en reproduction confirme le point de vue de Heymans (1996) qui conseille de mettre en reproduction des femelles ayant au moins $2000 \mathrm{~g}$. Le fait que plus de $70 \%$ des mortalités soient dues aux infections respiratoires ou gastro-intestinales et aux accidents montrent que la mortalité est principalement liée à l'environnement à travers le logement, l'alimentation et la conduite de l'élevage.

\section{CONCLUSION}

Au terme de l'étude sur l'évaluation des performances de reproduction des aulaco-

\section{BIBLIOGRAPHIE}

CIRAD. 2003. Elevage des aulacodes, informations légales. CIRADD. Montpellier, France. 12 p.

Deblay, S. 2002. Mémento de la reproduction des mammifères d'élevage. ENESAD. France.

FAO. 2001. La situation mondiale de l'alimentation et de l'agriculture. Organisation des Nations unies pour l'Alimentation et l'agriculture. Rome. p. 62-71.

FAO. 2002. La situation mondiale de l'alimentation et de l'agriculture. Organisation des Nations Unies pour l'Alimentation et l'agriculture. Rome. p. 3-15.

Heymans, J.C. 1996. L'élevage de l'aulacode (Tryonomys swinderianus). Cahier FAO (31). Rome. 79 p.

Mensah, G.A. 2000. Présentation générale de l'élevage d'aulacodes, historique et état de diffusion en Afrique. Actes du séminaire international sur l'élevage intensif du gibier a but alimentaire en Afrique. Libreville. Gabon. p. 4559. des en captivités dans les hautes terres de l'Ouest du Cameroun, les principales conclusions ont été les suivantes: La durée de gestation est légèrement plus longue et varie peu avec le numéro de mise- bas. La taille de la portée est supérieure à celle rapporté dans la littérature, cependant elle est suivie par une mortinatalité élevée, notamment en seconde mise-bas. Les poids à la naissance et au sevrage sont comparables aux données de la littérature et augmentent significativement avec le numéro de mise-bas. La mortalité post-natale des petits baisse à la seconde mise bas et avec le poids croissant de mise en reproduction. La morbidité et les mortalités seraient associées à diverses causes, les plus importantes étant les affections respiratoires, les maladies gastro-intestinales et les accidents. Les causes inconnues restent importantes.

Mensah, G.A. et M.R.M. Ekue. 2003. L'essentiel en Aulacodiculture. Réseau Rongeurs et Environnement. Cotonou. $160 \mathrm{p}$.

Nguema, M. et D. Edderai. 2000. La gestion de la reproduction en aulacodiculture. Actes du séminaire international sur l'élevage intensif du gibier a but alimentaire en Afrique. Libreville. Gabon. p. 60-66.

Schrage, R. et L.T. Yewadan. 1995. Abrégé d'aulacodiculture. GTZ № 251. Rossdorftz-Vert Ges. Allemagne. 103 p.

Siter, C.H., G.A. Mensah et C.F. Gall. 1991. Élevage d'aulacodes (Tryonomys swinderianus) pour la production de viande. World Anim. Rev., 69: 4449.

Van de Velde, M. 1991. Élevage des aulacodes au Zaire. Publication du service agricole $N^{\circ} 27$, AGCD. Bruxelles. Belgique. $90 \mathrm{p}$.

World Bank. 2002. African development indicators 2002. The World Bank. Washington D.C. USA. $376 \mathrm{p}$. 